\title{
Experimental Evaluation of Asymmetric QoS in IEEE 802.11g Wireless Networks
}

\author{
Qi Gang (William) Hu \\ School of ICT \\ Southern Alberta Institute of \\ Technology \& University of Calgary \\ Calgary, Canada \\ william.hu@sait.ca
}

\author{
Carey Williamson \\ Department of Computer Science \\ University of Calgary \\ Calgary, Canada \\ carey@cpsc.ucalgary.ca
}

\author{
Abraham O. Fapojuwo \\ Department of Electrical and \\ Computer Engineering \\ University of Calgary \\ Calgary, Canada \\ fapojuwo@ucalgary.ca
}

\begin{abstract}
Quality of Service (QoS) can be provided in a Wireless Local Area Network (WLAN) using the Enhanced Distributed Channel Access (EDCA) mechanism specified in IEEE 802.11e. However, 802.11e WLANs are not widely deployed and not all WLAN vendors implement the 802.11e mechanisms. In this paper, we propose and evaluate an asymmetric QoS solution, in which QoS support is provided only at the wireless Access Point (AP). We believe that this approach provides a practical solution for many cases where wireless clients may not support EDCA QoS. The feasibility of this solution is studied, using an experimental approach. A QoS testbed is designed and implemented using a centralized wireless controller and lightweight AP. The measurement results show that VLAN-based asymmetric QoS provides effective prioritization and excellent performance for high-priority traffic classes, including Voice over IP (VoIP) and TCP traffic, even during severe congestion conditions. Furthermore, this approach can be easily implemented using minimal equipment.
\end{abstract}

\section{Categories and Subject Descriptors}

C.2.1 [Computer-Communication Networks]: Network Architecture and Design - Wireless Communication.

\section{General Terms}

Performance

\section{Keywords}

WLAN QoS; 802.11e EDCA; Asymmetric QoS; VoIP over WLAN

\section{INTRODUCTION}

IEEE 802.11 Wireless Local Area Networks (WLANs) have been widely deployed in enterprise and campus networks over the past decade, and have become a fundamental component of the converged network infrastructure in enterprises. Today's WLAN environments need to support many different types of traffic, such as delay-sensitive voice, video streaming, and mission-critical traffic with specific Quality of Service (QoS) requirements. The provisioning of QoS in WLAN environments is challenging, and

Permission to make digital or hard copies of all or part of this work for personal or classroom use is granted without fee provided that copies are not made or distributed for profit or commercial advantage and that copies bear this notice and the full citation on the first page. To copy otherwise, or republish, to post on servers or to redistribute to lists, requires prior specific permission and/or a fee.

Q2SWinet'11, October 31-November 4, 2011, Miami, Florida, USA.

Copyright 2011 ACM 978-1-4503-0899-1/11/10...\$10.00. becoming increasingly important in many cases.

Most of today's WLANs use conventional IEEE 802.11 protocols, such as the Distributed Coordination Function (DCF). DCF is a contention-based Medium Access Control (MAC) protocol that uses Carrier-Sense Multiple Access with Collision Avoidance (CSMA/CA) to arbitrate channel access among the contending wireless stations. With 802.11 DCF, all stations associated with the same Access Point (AP) operate independently and share the channel bandwidth. Therefore, it is difficult to satisfy the low delay and jitter requirements of VoIP traffic over WLANs [1].

In order to support VoIP over WLANs, IEEE proposed Enhanced Distributed Channel Access (EDCA) in 802.11e, which is a QoS extension to legacy 802.11 DCF. With EDCA, high priority traffic has a higher probability of being sent than low priority traffic. In other words, a station with high priority traffic waits less, on average, than a station with low priority traffic. This is accomplished by using a smaller Contention Window $(\mathrm{CW})$, and shorter Arbitration Inter-Frame Space (AIFS) values for higher priority packets. In addition, EDCA provides sustained access to the channel in a contention-free fashion for a period called a Transmit Opportunity (TXOP). A TXOP is a bounded time interval during which a station can send as many frames as it wishes, provided that the duration of the TXOP is not exceeded. If a frame is too large to fit within a single TXOP, it is fragmented into smaller frames. The use of TXOPs ameliorates the problem of low-rate stations monopolizing use of the channel in the legacy 802.11 DCF MAC [8].

\subsection{Problem Statement}

Many research efforts have studied the problem of supporting Voice over IP (VoIP) on 802.11 WLANs, but few works focus on practical implementations [3]. In a converged network environment, WLANs process many types of traffic. It is important to investigate and evaluate the performance of the actual wireless QoS implementations based on the widely deployed wireless equipment and wireless adapter cards. While the 802.11e EDCA standard has been adopted by major wireless vendors, the EDCA QoS configuration is still not widely supported on wireless adapters, PCs, and laptops. The challenges of provisioning QoS over real wireless LAN include:

- Is it possible to implement WLAN QoS on the AP only? (i.e., Asymmetric QoS)

- How would the network perform if QoS is only configured on the AP? 


\subsection{Motivation}

802.11 wireless network technologies are advancing so fast that many new protocols are not fully adopted in the same way among vendors, such as 802.11e.

There are some limitations that need to be considered when implementing QoS on WLAN. The following paragraphs provide the main reasons motivating us to conduct this asymmetric wireless QoS performance study.

First, we conducted a technical survey of the wireless computers in a large campus WLAN, and found that the majority of wireless adapter cards in the PCs and laptops used by faculty and students do not support 802.11e EDCA QoS parameter configurations. This observation raises an interesting question concerning wireless QoS deployment in the existing wireless networks: Is it possible to obtain most of the benefits of WLAN QoS by implementing QoS only on the AP (since the wireless clients do not support EDCA QoS)? This motivates us to propose the concept of Asymmetric QoS solution in this paper. It would provide a practical and cost effective solution for the widely deployed wireless networks if the proposed Asymmetric QoS works and provides suitable QoS.

Second, we conducted a literature review, and found no research work focused on asymmetric QoS implementation. 802.11 g/n are the major WLANs deployed in enterprise campus today, most APs are 802.11e EDCA certified to support Wireless QoS, however the actual EDCA capabilities supported are quite different. For instance, some APs support traffic classification at protocol level; others only allow VLAN based classification, such as Cisco Aironet $\mathrm{C} 1240$ used in this research. Therefore investigating QoS performance for VLAN-based traffic classification would be beneficial for users using similar equipment.

Third, one popular VoIP over WLAN solution is using softwarebased phones. For example, Cisco IP Communicator (CIPC) is a VoIP softphone for Windows PCs. We would like to investigate the QoS impact on performance of this software-based phone running on regular wireless Windows PCs.

Lastly, not only the QoS of voice traffic can be evaluated in this experiment, but also the QoS of other traffic such as TCP. In this paper, the QoS interplay between different traffic types can be evaluated by associating them with respective VLANs.

\subsection{Contributions}

The primary contribution of this work is the concept of asymmetric QoS implementation. This approach is attractive because it requires minimal wireless equipment, and provides suitable QoS support for delay-sensitive and mission-critical traffic. As a secondary contribution, this paper presents the design and implementation of a VLAN-based wireless QoS evaluation testbed, which we use to evaluate our proposed solution experimentally. The measurement results demonstrate that the asymmetric QoS is a feasible and robust solution for WLANs with asymmetric traffic. Specifically, supporting QoS only on the AP can provide effective WLAN QoS for high priority traffic.

\section{Related Work}

With the rapid growth in WLAN popularity, wireless QoS has attracted a lot of research attention. IEEE 802.11e proposed EDCA wireless QoS standards that act as a guideline for VoIP over WLAN. Today's WLANs support data rates approaching $300 \mathrm{Mbps}$. VoIP has already gained widespread use based on its functionality and low cost for deployment. Combining WLAN and VoIP together is becoming an attractive converged solution for enterprise networks.

Although modern WLANs offer high data rates, bandwidth alone is not always sufficient to accommodate the needs of VoIP applications. For instance, the actual capacity of WLAN to accommodate VoIP calls may be far less than the theoretical calculation. Jeong et al. [1] present research on the VoIP call capacity analysis. While $802.11 \mathrm{~b}$ can theoretically accommodate 85 calls, the poor efficiency of the MAC-layer protocol limits this to only 5 calls in practice. Equation (1) gives the expression for calculating $N_{P H Y}$, the maximum number of calls supported, taking into account the overheads on data link and physical layers [1]: The Maximum \# of Calls at Physical layer:

$N_{P H Y}=\frac{1}{2 k\left(T_{D I F S}+T_{B O}+T_{S I F S}+T_{A C K}+T_{P H Y}+T_{L 3}\right)}$

where $k$ denotes the frame rate of the voice codec, set to 50 frames per second for G.711. $T_{L 3}$ denotes the time taken to transmit a layer 3 packet; the remaining components $T_{D I F S}, T_{B O}, T_{S I F S}$, and $T_{A C K}$ are the DCF timers (see Figure 1) and $T_{P H Y}$ is the layer 1 overhead. According to eqn. (1) layer 2 contributes the most overhead to the overall frame transmission time. Jeong et al. [1] proposed an algorithm to implement MAC-layer frame aggregation, which improved the VoIP call capacity by $300 \%$. Because of high WLAN overhead, it is obviously necessary to implement QoS on WLAN to support efficient traffic delivery for converged networks.

A practical WLAN QoS solution is proposed in [3]. This work discussed EDCA parameter tuning on both AP and WiFi phones. Their analysis found that the AP has higher probability of gaining channel access than wireless clients given the same EDCA parameters on both sides. Basically, the AP does not need more aggressive EDCA parameters than clients [2]. That means the AP has the advantage of winning the wireless channel access, and it contributes major impact for WLAN QoS when both parties are using the default EDCA configurations. This work [2] determined sets of optimal EDCA parameters for AP and clients based on simulations and compared the performance with default EDCA values in experiments.

Achieving strict QoS for high priority traffic may sacrifice the services for other lower priority traffic due to limited bandwidth. To balance the QoS services for different types of traffic, and provide proportional services for other ranked traffic flows, Lee et al. [7] proposed a Differentiated Service-EDCA (DS-EDCA) model to provide strict QoS for voice traffic, by managing fairness for other traffic flows using weighted fair parameters. With DS-EDCA, the resource is first allocated to flows of higher priority. The remaining bandwidth is then shared proportionally among the other service priorities according to their assigned weights. A hierarchical link sharing model for IEEE 802.11e WLANs is proposed, which is very useful in downlink and uplink traffic sharing. Their results are verified using simulation.

Most WLAN QoS researches focus on VoIP capacity analysis and improvements [2], [5], [10]. Many works have been done in terms of EDCA parameter optimization, with QoS analytical modeling and simulations as the major approaches used. These works provide a solid foundation from which to explore more advanced WLAN QoS strategies, such as class-based queuing and weighted fair-queuing techniques over WLAN. However, few works have been conducted to evaluate the QoS performance over 802.11g 
network using commercially available Windows PCs and vendorspecific equipment such as Cisco Aironet wireless equipment.

\section{Background: 802.11 Wireless QoS}

\subsection{11 Wireless Network DCF MAC}

IEEE $802.11 \mathrm{~b} / \mathrm{g}$ standards are the popular WLAN protocols deployed in enterprise campus networks. They use the same media access control mechanisms: DCF, RTS/CTS, and PCF. The distributed coordinated function (DCF) and RTS/CTS are commonly used in most cases.

Wireless frame transmission is controlled by DCF and RTS/CTS mechanism [14]. Figure 1 [8] shows the 802.11 DCF media access control mechanism. DCF is composed of two components: Interframe Space (IFS) and Contention window CW, which is determined based on $\left(C W_{\min }, C W_{\max }\right)$, where $C W_{\min }$ and $C W_{\max }$ are respectively the minimum and maximum contention window, whose values depend on the access category, as shown in Table 1.

The IEEE 802.11 currently defines three inter-frame spaces:

- $\quad$ Short inter-frame space (SIFS) $10 \mu \mathrm{s}$. Important frames such as acknowledgement and management frames wait SIFS before transmission.

- $\quad$ Point (coordination function) inter-frame space (PIFS) SIFS $+1 \times$ slot time $(20 \mu \mathrm{s})=30 \mu \mathrm{s}$. only used in polling system.

- Distributed (coordination function) inter-frame space (DIFS) $50 \mu \mathrm{s}$, SIFS $+2 \times$ slot time $=50 \mu \mathrm{s}$.

Data frames wait DIFS before starting the random backoff procedure [14][10].

When a data frame is ready to be sent, the sender generates a random backoff number $n$ uniformly between 0 and $\mathrm{CW},(\mathrm{CW}=$ $C W_{\min }$ initially) and checks the channel to ensure it is free for DIFS interval, then starts backoff for $n \times$ slot time if the channel remains free throughout the backoff period, data is sent after the backoff period; $\mathrm{CW}=C W_{\min }$ after each successful transmission. If the channel is busy during the backoff period, the wireless device pauses the backoff counter and resumes it DIFS after the end of frame again. Following the $i^{\text {th }}(i \geq 1)$ unsuccessful transmission attempt, the random backoff number $n$ is selected from a uniform process repeats until the maximum number of retransmission attempts $m$ is reached, and the transmission is aborted in this case.

\subsection{Wireless QoS Mechanism}

The purpose of QoS is to provide required network transmission services for applications, by controlling the delay, jitter, and packet loss to be within pre-defined values.

Wireless QoS is provisioned by controlling the DIFS and random backoff time. 802.11e EDCA defines different arbitration interframe space (AIFS) and contention window parameters for different traffic categories, so that the high priority traffic waits less than low priority traffic. Statistically, high priority traffic has a higher chance to be transmitted, especially during heavy load conditions.

Table 1 shows the EDCA parameters defined for four traffic Access Categories (ACs): AC_VO; AC_VI; AC_BE; AC_BK. [13] The arbitration inter-frame space time interval - AIFS can be calculated using the following formula:

$$
\begin{aligned}
& \text { AIFS }[\mathrm{AC}]=[\mathrm{SIFS}]+\mathrm{AIFSN}[\mathrm{AC}] \times[\text { aslotTime }] \\
& (\mathrm{AIFSN}[\mathrm{AC}]>=2)
\end{aligned}
$$

Table 1. Default EDCA Parameters for each AC

\begin{tabular}{|lcccc|}
\hline \hline \multicolumn{1}{|c}{ AC } & CWmin & CWmax & AIFSN & Max TXOP \\
Background (AC_BK) & 31 & 1023 & 7 & 0 \\
Best Effort (AC_BE) & 31 & 1023 & 3 & 0 \\
Video (AC_VI) & 15 & 31 & 2 & $3.008 \mathrm{~ms}$ \\
Voice (AC_VO) & 7 & 15 & 2 & $1.504 \mathrm{~ms}$ \\
Legacy DCF & 15 & 1023 & 2 & 0 \\
\hline
\end{tabular}

where AIFSN is the AIFS number. IEEE 802.11 wireless communication uses a shared half-duplex mechanism, in which all devices are competing to gain channel access. Only one device can transmit in one direction at a time. If the channel is busy, then other devices have to backoff random amount of time, which is controlled by EDCA-defined parameters in Table 1. Since the high priority category waits for a shorter backoff time, it tends to get served more frequently.

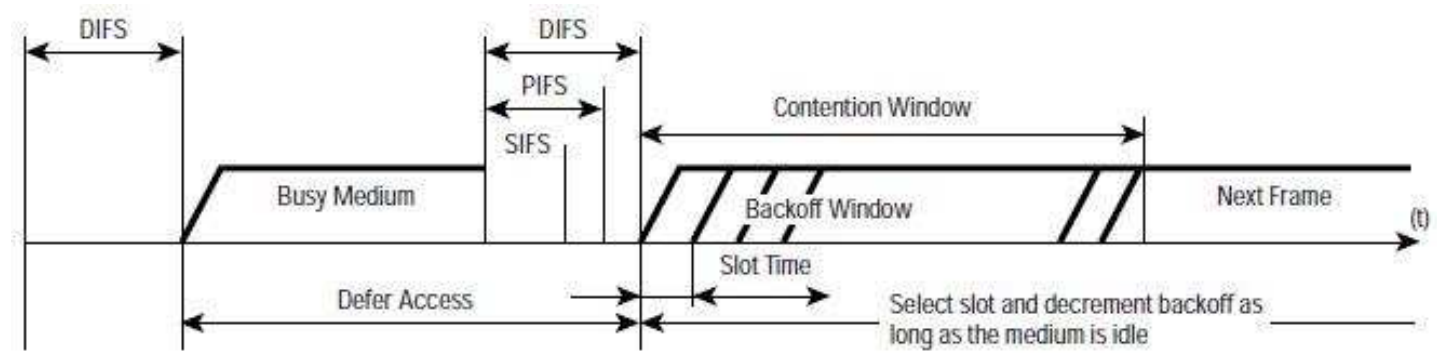

Figure 1. IEEE 802.11 DCF Timing Illustration [8]

distribution in the range $\left[0, C W_{i}-1\right]$ where $C W_{i}$ is the contention window size at the $i^{\text {th }}$ retransmission attempt, calculated by: $C W_{i}=2^{i} C W_{\min }$, such that $C W_{\min } \leq C W_{i} \leq C W_{\max }$ and $1 \leq i \leq m$, where $m$ is the maximum number of retransmissions satisfying $C W_{\max }$. That is, $m=\log _{2}\left(C W_{\max } / C W_{\min }\right)$. Once the $C W_{i}$ reaches $C W_{\max }$ it remains at the value of $C W_{\max }$ until it is reset by a successful transmission. The contention window ascending

\subsection{Wireless Traffic Flow Pattern Analysis}

There is typically asymmetry in the traffic flow pattern in an access network, and the same is true in WLAN environments. The $\mathrm{AP}$ is the central multiplexing point in a wireless network. It aggregates traffic to and from clients, and it may become the bottleneck of the wireless network, since it is involved in the transmission of approximately half of the packets in the WLAN.

Consider a simple example. Suppose there are two clients associated with an AP, each client makes a call to wired IP phone, 
the bandwidth required for each call is $128 \mathrm{kbps}$ (G.711) in each direction. The AP will need bandwidth of $512 \mathrm{kbps}(4 \times 128 \mathrm{kbps})$ to serve the two clients. If the two pairs of calls are made between four wireless clients, the AP would need a bandwidth of 1024 kbps $(8 \times 128 \mathrm{kbps})$.

The bandwidth requirements for $\mathrm{AP}, B_{A P}$, to serve $c$ clients with bandwidth requirement $\beta$ for each client in each direction can be estimated as follows:

$$
B_{A P}=2 c \beta
$$

So if multiple clients send large volumes of traffic over wireless network creating congestion condition, the AP would become congested before the clients, assuming the AP and the clients are operating at the same data rate.

To analyze the QoS effect, we need to look at the downstream and upstream traffic separately.

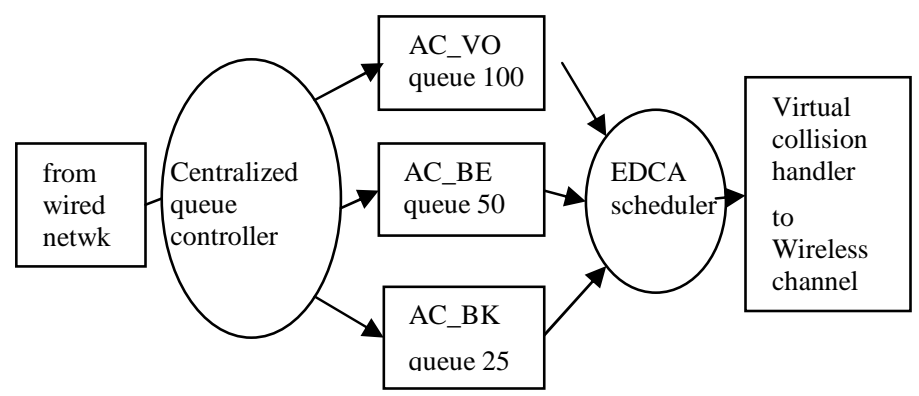

Figure 2. AP QoS Handling Illustration for Downstream Traffic

Downstream traffic is the traffic flowing from AP to wireless client. This direction of traffic flow dominates the major QoS performance because the downstream traffic is originated from high-speed wired networks flowing towards slower speed wireless clients through APs. In most networks, wireless clients download configured to meet the traffic QoS requirements. If the downstream buffer has a long queue, this could result in higher delay and jitter for VoIP packets. Taking the above factors into account, the downstream link tends to be more congested than the upstream link, therefore the QoS applied in downstream direction becomes more important and it has more effective impact than the upstream QoS. Figure 2 illustrates the QoS process for downstream traffic [7].

Three priority categories are configured on AP using VLANbased EDCA QoS mechanism. The packets from wired network are classified into their respective $\mathrm{AC}$ queue based on the destination VLAN. The AC_VO is assigned the shortest AIFS backoff interval, and relatively larger queue size, so it has the highest opportunity to be transmitted during the channel access competition. On the other hand, AC_BK is assigned the longest AIFS backoff interval and smallest queue size, so it has the lowest opportunity to get transmitted. Also, since its queue may fill earlier than any other categories, more packets from this category may get dropped when congestion occurs. AC_BE has medium AIFS backoff interval, so that it receives service between the other two categories.

Upstream traffic refers to the traffic flowing from wireless client towards APs. $802.11 \mathrm{~g}$ wireless LAN is much slower than the wired Ethernet, so congestion won't happen for upstream traffic transiting from the wireless network to the wired network. Most wireless clients download more than what they upload [12]. Considering these factors, the limitation of wireless adapters not supporting the EDCA parameter configuration would impose less impact for the wireless QoS performance. According to the above traffic flow pattern analysis, asymmetric QoS is a feasible solution for wireless networks with asymmetric traffic pattern.

\section{EXPERIMENT DESIGN AND IMPLEMENTATION}

The experimental QoS measurement testbed is built to conduct the wireless VLAN-based QoS performance evaluation. Figure 3

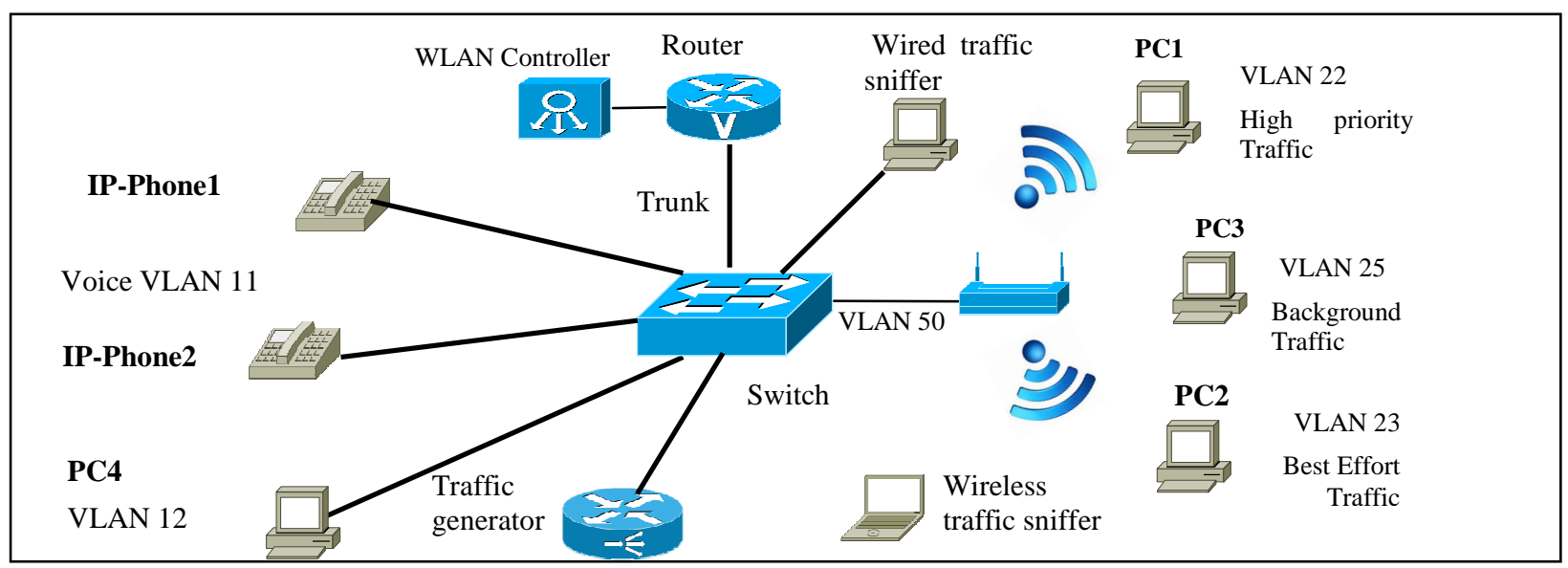

Figure 3. Wireless QoS Measurement Topology

much more content than they upload.

According to a comprehensive campus WLAN measurement study conducted by Mahanti et al. [12], the overall ratio between downstream and upstream traffic is typically 2-to-1. This asymmetric traffic pattern measured on real campus wireless networks provides grounds for our idea to support asymmetric QoS implementation. Furthermore, the AP aggregates all the downstream traffic for all clients, and needs to be properly shows the testbed topology.

The lab uses Cisco centralized WLAN controller module which is able to support 16 lightweight APs. We configured one Cisco Aironet 1240 AP with three wireless VLANs to support three traffic classes for QoS performance test. VLAN 22 is assigned high priority to accommodate voice or mission-critical traffic. VLAN 23 is used for best-effort traffic. VLAN 25 is designated for background traffic. 
In order to get an overall picture of the bandwidth utilization by different types of traffic, a wired monitor station is used to capture network traffic from the trunk port on the switch. A wireless sniffer running BackTrack Linux [15] is set up in monitor mode to perform the wireless traffic measurement over different wireless VLANs.

QoS prioritizes mission-critical and delay-sensitive traffic so that these traffic classes get more chance to be forwarded with less delay and jitter. However, QoS is designed to manage the congestion situation. When network traffic volume is light, and the probability of wireless channel contention is very low, it is rare to observe QoS problems among traffic classes. For this reason, a traffic generator TGEN is used to stress the network. This is configured by using Cisco router running special traffic generator operating system Pagent to generate background traffic, creating controlled congestion conditions for the QoS evaluations. TGEN generates background traffic that consists of Telnet, FTP, and HTTP packets with random packet size between 16 1500 bytes. Different data rates from 800 to 2500 packets/sec are used in the experiment to create appropriate congestion conditions for different traffic measurements so that the best comparison can be obtained.

Three VLANs are created to accommodate three traffic categories: AC_VO; AC_BE and AC_BK. To compare the QoS performance we first assign all VLANs to AC_BE category with the best effort service and measure the baseline performance results. Then we re-assign VLAN 22 to AC_VO class with the highest priority, put VLAN 23 into AC_BE category providing best effort QoS, assign VLAN 25 to AC_BE (for VOIP test) or AC_BK (for TCP test) as background traffic with different QoS category, and perform the traffic measurement again to compare and analyze the results.

\section{EXPERIMENTAL RESULTS}

In this section, the traffic measurement results are carefully selected from 50 trace files (over 10 Gigabytes in total) captured from wireless VLAN 22, 23, 25 and wired trunk port. In order to test a wide range of operating conditions, the traffic measurements are conducted with different background traffic rate: 800,1000 , $1200,1500,2000,2500$ packets/sec. Only a subset of these results is presented in the paper, however. Specifically, we select results that best illustrate the QoS impact on voice and TCP traffic. For VoIP QoS evaluation, the delay, jitter, and packet loss metrics are used to compare the performance results. For TCP traffic, TCP sequence number plots are used to illustrate connection progress over time, in addition to overall average throughput.

\subsection{VoIP Results}

\subsubsection{Baseline Results with No QoS}

Figure 4 shows the average performance measurement results from multiple tests for voice conversations between softwarebased phones on PC1 and PC2, and IP phones, all without QoS. The background traffic is generated by traffic generator TGEN, which generates three traffic streams (Telnet, HTTP and FTP) at two rates: 800 and 1000 packets/sec. The two different rates create different congestion conditions. All three VLANs (including VLAN 25 for background traffic) are given the same traffic access category, namely AC_BE.

With 800 packets/sec downstream background traffic, there is distinct uneven packet loss and jitter observed between downstream link and upstream traffic. The downstream link had
20-30\% packet loss, with a jitter of 10-15 ms, while the upstream traffic had no packet loss and lower jitter of $4 \sim 7 \mathrm{~ms}$.

PC1 and PC2 cannot initiate a call towards the wired IP Phones when the background traffic rate increases to 1000 packets/sec; however IP Phones can initiate the call back to PC1 or PC2. Once the call is placed successfully, the softphone experienced very poor voice quality. The recipient heard jerky and partial words, and could hardly understand sentences. On the other hand, the voice quality on IP Phones is fair, smooth, and clear. Both VLANs have no packet loss for upstream traffic.

These results make sense for the specific traffic scenario considered. Because the traffic generator generates one way downstream traffic flowing from AP to wireless client PC3, the downstream voice traffic has to compete with this heavy background traffic to reach $\mathrm{PC} 1$ and $\mathrm{PC} 2$. AP maintains fairness [14] while distributing traffic to PC1, PC2, and PC3. This heavy background traffic can overflow the AP's downstream buffer [7][8], with the Tail-Drop mechanism discarding subsequent voice and TCP packets. If the TCP packets were originated from a normal Internet application, such as an FTP server, the TCP congestion control mechanism would slow down the transmission rate. However, here the TCP packets are generated in an openloop (fixed-rate) fashion by the traffic generator, which does not respond to any packet drops. Instead, it maintains its predefined transmission rate. As a result, the downstream wireless client PC1 and PC2 experienced a lot of packet losses and jitter due to intense competition on downstream link. Therefore, the downstream client on the softphone heard really poor voice quality, while the upstream IP phone still had pretty good voice quality.

Both VLAN 22 and 23 perform similarly without QoS. Figure 4 and Figure 5 exhibit the average results from five separate measurements in the same day.

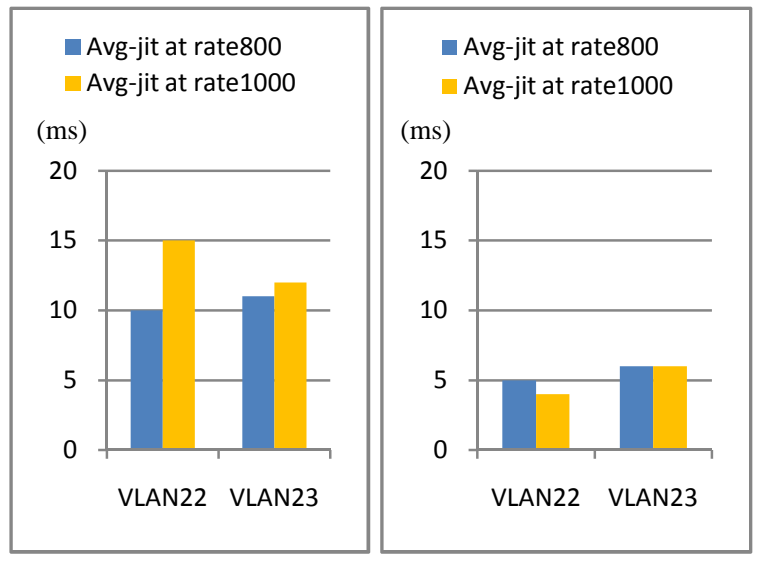

Figure 4. Downstream (Left) and Upstream (Right) Average jitter without QoS.

\subsubsection{Results with Asymmetric QoS}

In this case, VLAN-based QoS on wireless network is enabled by assigning VLAN 22 the highest EDCA category (AC_VO) to accommodate high priority voice traffic. VLAN 23 is given the medium EDCA category (AC_BE). The background traffic VLAN 25 is also given medium category (AC_BE) to compete with VLAN 23 voice traffic directly, so that the QoS implication on voice traffic over dedicated voice VLAN 22 and normal VLAN 23 which has voice traffic and background traffic mix can be compared. The test scenario is otherwise the same as before. 
With QoS enabled, VLAN 22 and VLAN 23 receive differentiated channel access probability to deliver the call with different jitter and packet loss when the background traffic rate is 800 packets/sec.

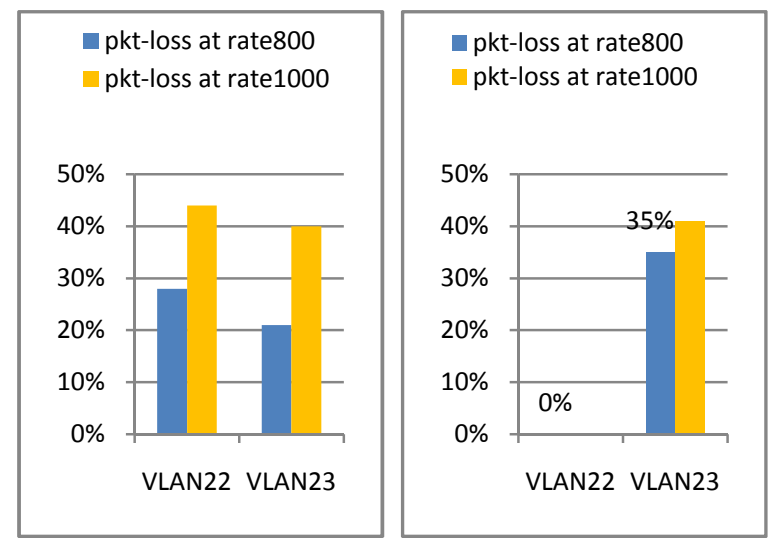

Figure 5. Downstream packet loss without QoS (Left), with QoS (Right).

Figures 5 and 6 show the measurement results for average jitter and packet loss between the two EDCA categories. Surprisingly, the phone call on VLAN 22 becomes fairly clear in both directions with QoS enabled when the background traffic rate is 800 packets/sec; the phone call can be initiated from either side. This result demonstrates the effectiveness of EDCA wireless QoS function, even if it is only configured on the AP.
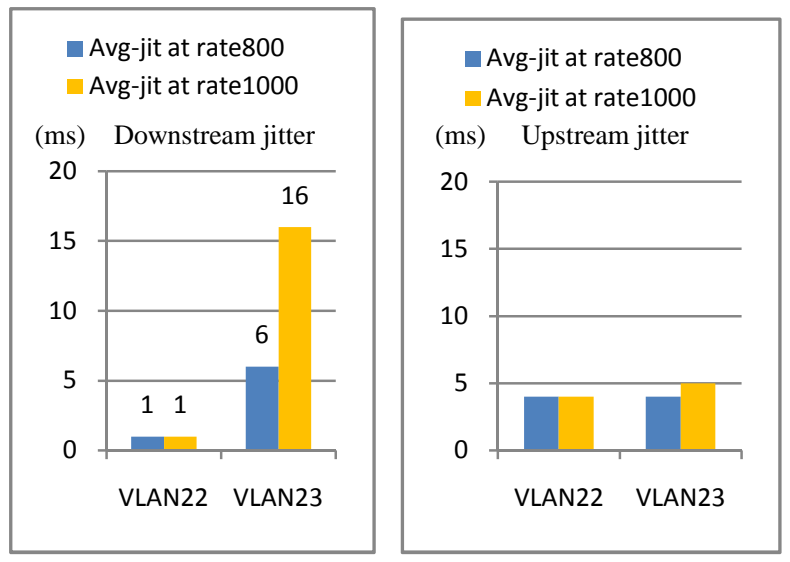

Figure 6. The Downstream (Left) and Upstream (Right) Average jitter with QoS

With background traffic rate at 800 packets/sec, the traffic measurement shows distinct results between VLAN 22 and VLAN 23 in Figures 5 and 6 . The voice traffic on VLAN 22 (AC_VO) received excellent downstream service with zero packet loss and minimal jitter of $1 \mathrm{~ms}$. The downstream voice traffic on VLAN 23 (AC_BE) still experienced 35\% packet loss (Figure 5, right) and $6 \mathrm{~ms}$ jitter because it is competing with the background traffic on VLAN 25 with the same priority category.

By increasing the background traffic rate up to 1000 packet/sec to exacerbate the congestion condition, the QoS provisioning capability on VLAN 23 is pushed up to the limit due to severe network congestion. The voice call on VLAN 23 (AC_BE) is unsustainable due to high packet loss (42\%). Its jitter also grows to $16 \mathrm{~ms}$. It becomes difficult to establish a call from PC2 to IP Phone. The call might be dropped in a few minutes even if the call was initiated from the other side. On the contrary, it is observed from Figures 5 and 6 that VLAN 22 performs excellently with no packet loss and $1 \mathrm{~ms}$ jitter.

Figure 6 also indicates that the upstream link maintains consistent small variation in average jitter between the two ACs with $0 \%$ packet loss (not shown on the graph). This is because the upstream traffic volume is much lighter than AP's capacity, so no packet loss occurs. QoS is not available on the PCs in this experiment. Since the AP treats all upstream traffic fairly, therefore the upstream average jitters from both VLANs (two ACs) are consistent.

\subsection{TCP Results}

\subsubsection{Baseline Results with No QoS}

All three VLANs (VLAN 22, 23, 25) are assigned the default EDCA QoS category (AC_BE). The traffic generator TGEN sends background traffic on VLAN 25 at rate of 800 packets/sec, in the meantime FTP downloading from and to both PC1 (VLAN 22) and PC2 (VLAN 23) are measured.

With heavy downstream background traffic volume, PC1 and PC2 had very low FTP download rate $(8 \mathrm{~KB} / \mathrm{s})$ from PC4, see Fig. 7. Sometimes the downloading timed out due to congestion. On the other hand, both PCs can smoothly upload files to PC4, although still with slow uploading speed of $76 \mathrm{~KB} / \mathrm{sec}$ (Figure 8 ).

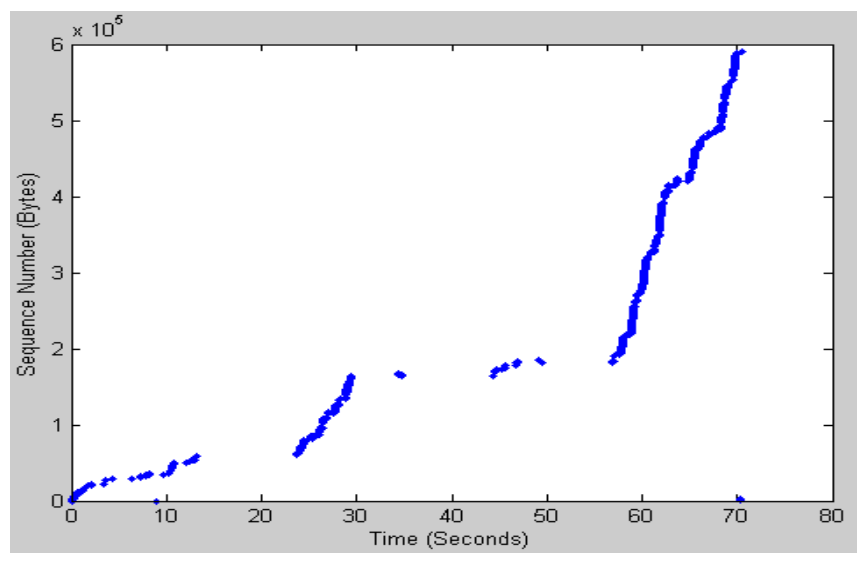

Figure 7. Downstream TCP Performance without QoS

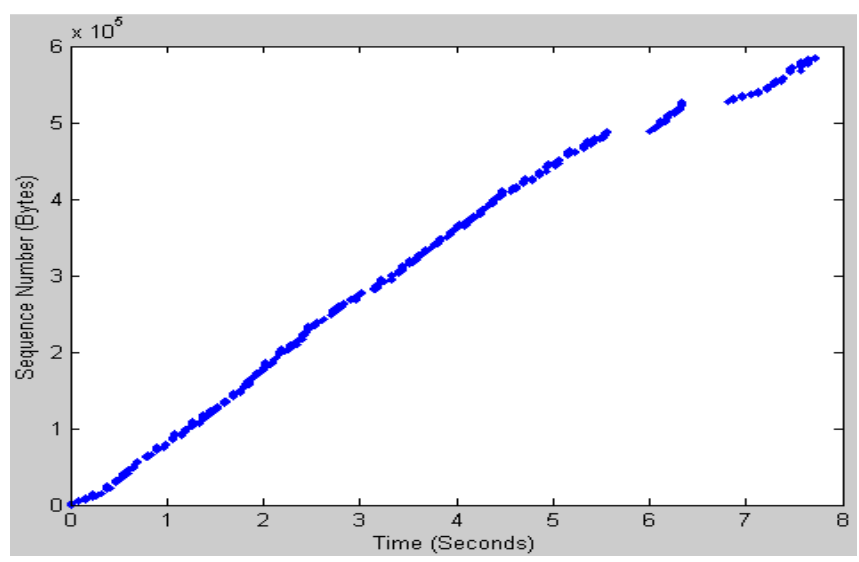

Figure 8. Upstream TCP Performance without QoS

The poorer downstream performance shown in Fig. 7 is because the downstream FTP traffic has to compete with heavy background traffic to reach $\mathrm{PC} 1$ and $\mathrm{PC} 2$. The AP maintains fairness while distributing traffic to $\mathrm{PC} 1, \mathrm{PC} 2$ and $\mathrm{PC} 3$. The 
background traffic sent at rate 800 packets/sec and FTP downloading traffic go to the same AC_BE queue which could result in the queue overflow, hence tail drop mechanism drops subsequent FTP packets and background TCP packets. The background TCP packets do not respond to any packet drops, but maintains a constant transmission rate. However, the TCP protocol of the real FTP streams reacts to the packet loss by using slow start, congestion avoidance, and retransmission timeout mechanisms to reduce the FTP downloading rate. As a result, the downstream FTP traffic experiences long delay or even being disconnected.

We can see there are a lot of packet losses, retransmissions, and timeout events in the two graphs. The significant timeout activities can be identified by the gaps in the curve. TCP doubles the retransmission timeout interval due to consecutive retransmission failures resulting from the severe congestion condition. Fig. 7 (The downstream graph) has many more gaps than Fig. 8 (The upstream graph). The TCP congestion window size is very small because of frequent packet losses.

The main reason causing the retransmissions and timeout events for the upstream traffic is because the ACKs from PC4 to PC1 and PC2 are lost due to heavily congested downstream link. Overall upstream transfer is ten times faster than the downstream due to the heavy downstream background traffic.

\subsubsection{Results with Asymmetric QoS}

In this case, the wireless QoS is implemented by assigning VLAN 22 to high priority category (AC_VO) to simulate mission-critical traffic. VLAN 23 is given Best Effort category (AC_BE). VLAN 25 is given the lowest category (AC_BK ). FTP transfers from and to VLAN 22 (PC1) and VLAN 23 (PC2) are evaluated while the traffic generator is sending the background traffic to $\mathrm{PC} 3$ at two different rates of 800 and 2500 packets/sec.

With the above configuration, the QoS effects are highly evident. The FTP processes on VLAN 22 and VLAN 23 can both upload the same file at almost the same speed $(420 \mathrm{~KB} / \mathrm{sec})$; there is not much difference between their performances because QoS is not implemented for upstream link, and the background traffic is flowing downstream, furthermore QoS allocates a shorter AIFS interval for TCP ACKs to come back to these two VLANs smoothly, so that allow both VLAN 22 and 23 to upload files.

More importantly, QoS enabled both PC1 and PC2 to download the files from $\mathrm{PC} 4$ with higher transfer rate than uploading in spite of competing with background traffic. $\mathrm{PC} 1$ achieved the speed of $679 \mathrm{~KB} / \mathrm{sec}$, and PC2 got $423 \mathrm{~KB} / \mathrm{sec}$. This indicates that EDCA wireless QoS is functioning effectively compared to the scenario without QoS.

In order to observe the differentiated QoS performance among the two VLANs in both directions, we increase the background traffic rate up to 2500 packets/sec to exacerbate the congestion condition to a more severe level.

With heavier background traffic volume the QoS performance for the two VLANs in the upstream directions are differentiated as well. The uploading rates for PC1 (VLAN 22) and PC2 (VLAN 23) are measured as $369 \mathrm{~KB} / \mathrm{sec}$ and $337 \mathrm{~KB} / \mathrm{sec}$ respectively. See Figures 11 and 12. FTP downstream maintains similar performance as before. Figure 9 and Figure 10 exhibit the downstream FTP performance.

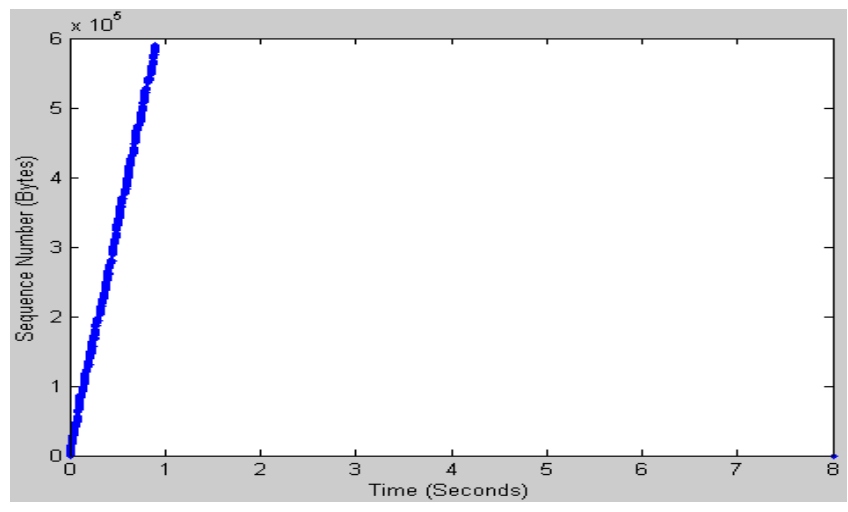

Figure 9. VLAN 22 Downstream TCP Performance with QoS

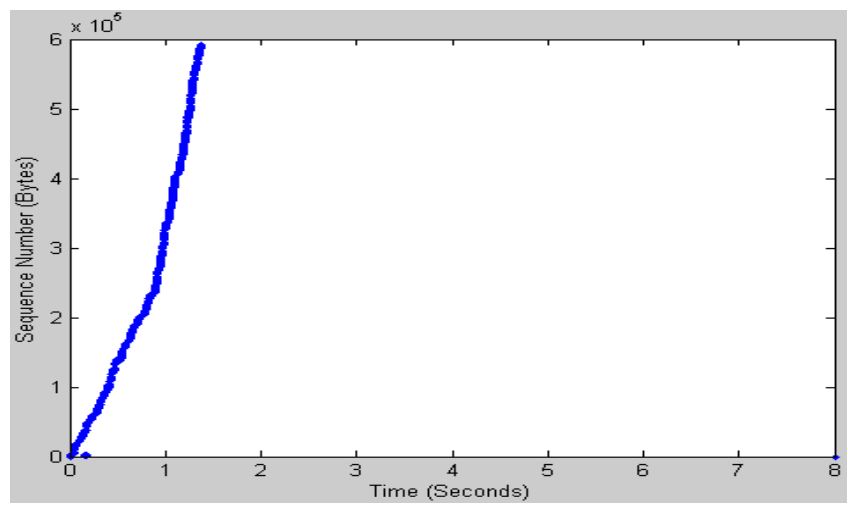

Figure 10. Vlan 23 Downstream TCP Performance with QoS

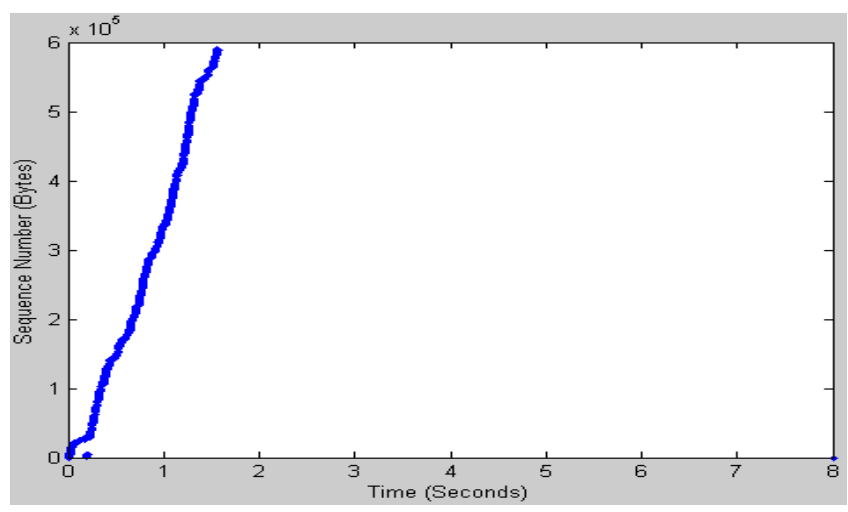

Figure 11. VLAN 22 Upstream TCP Performance with QoS

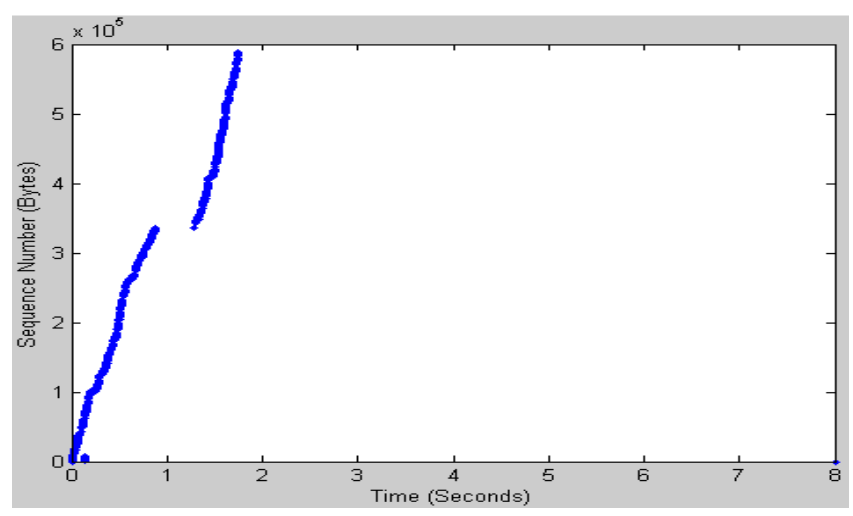

Figure 12. VLAN23 Upstream TCP Performance with QoS 
After increasing the background rate to 2500 packets/sec, the crowded downstream link causes some TCP ACK losses for upstream traffic which triggers TCP congestion control mechanism to slow down the FTP uploading rates. However, the downloading rates for both VLANs still maintain almost the same range. It means that ACK loss has more sensitive impact on TCP transfer rate than the actual data packet loss.

By comparing the TCP graphs in Fig. 7 with Figs. 9 and 10, a significant improvement on the downstream transfer rate can be seen after enabling QoS. PC1 and PC2's downloading speed increased by eighty and fifty five times respectively. The packet loss and retransmission activities are reduced dramatically. A distinct QoS effect can be observed by comparing Fig. 8 with Fig. 11 and Fig. 12. The uploading speeds from PC1 and PC2 to PC4 were increased by seven and five times even though QoS is only implemented on AP for downstream link.

According to Table-1 and Eqn. (2), the initial arbitrary inter-frame space for VLAN 25, VLAN 23, and VLAN 22 are estimated as $150 \mu \mathrm{s}, 70 \mu \mathrm{s}$, and $50 \mu \mathrm{s}$, respectively.

As the network gets congested, the QoS mechanism on AP controls the backoff time for each VLAN traffic based on the above initial AIFS. The lower priority AC (VLAN 25 background traffic) frequently encounters a busy channel, so the AIFS value of the lower priority AC increases exponentially before higher priority ACs when the retry counter is reached. As a result, higher priority traffic categories receive much better service at the cost of degrading service for lower priority traffic category. According to this, the downloading traffic from $\mathrm{PC} 1$ and $\mathrm{PC} 2$ has been prioritized accordingly based on their ACs. So they are able to maintain the downloading rates as the background traffic volume (AC_BK) increases.

For upstream traffic, the AIFS backoff time mainly affects the TCP ACKs, which indirectly influence the overall upstream FTP transfer speed. Since ACK loss has more sensitive impact on TCP traffic rate than data packet loss, therefore the uploading rate for PC1 and PC 2 were decreased by $12 \%$ and $20 \%$ respectively while the background traffic volume increased from 800 to 2500 packets/sec because of more ACK losses.

\section{CONCLUSION}

An asymmetric VLAN-based 802.11 wireless QoS solution is proposed in this paper. This may be a feasible and cost effective solution for wireless networks with asymmetric traffic pattern according to the analysis on downstream and upstream wireless traffic pattern and characteristics. Experimental approach is used to evaluate the performance of the proposed QoS implementation. The measurement and analysis results show that the asymmetric QoS provides distinct performance for different priority traffic categories. EDCA parameter has effective prioritization control over VLAN-based traffic categories even though the QoS is only supported on AP over a wireless network. The asymmetric QoS improves the transfer rate for both downstream and upstream TCP traffic even under severe congestion condition. Furthermore this approach provides a practical wireless QoS solution that is easy to deploy.

\section{ACKNOWLEDGMENTS}

The authors thank the Q2SWinet 2011 reviewers for their insightful feedback on the initial version of this paper. Financial support for this research was provided by SAIT (Southern Alberta Institute of Technology), iCORE
(Informatics Circle of Research Excellence) and NSERC (Natural Sciences and Engineering Research Council).

\section{REFERENCES}

[1] Y. Jeong, S. Kakumanu, C. Tsao, and R. Sivakumar, "VoIP over Wi-Fi Networks: Performance Analysis and Acceleration Algorithms", IEEE Conference on Broadband Communications, Networks, and Systems (BroadNets), 2007.

[2] S. Harsha, A. Kumar, and V. Sharma, "An Analytical Model for Performance Evaluation of Multimedia Applications over EDCA in an IEEE 802.11e WLAN", Wireless Networks Vol. 16, No. 2, pp. 367-385, February 2010.

[3] J. Lee, W. Liao, J. Chen, and H. Lee, "A Practical QoS Solution to Voice over IP in IEEE 802.11 WLANs", IEEE Communications, Vol. 47, No. 4, pp. 111-117, April 2009.

[4] P. Wu, J. Chen, Y. Tseng, and H. Lee, "Design of QoS and Admission Control for VoIP Services over IEEE 802.11e WLANs", Journal of Information Science and Engineering, Vol. 24, No. 4, pp. 1003-1022, July 2008.

[5] D. Gao, J. Cai, C. Foh, C. Lau, and K. Ngan, "Improving WLAN VoIP Capacity Through Service Differentiation", IEEE Transactions on Vehicular Technology, Vol. 57, No. 1, January 2008.

[6] C. Williamson, "Wireless Internet: IEEE 802.11b", CPSC 641 Lecture Notes, Department of Computer Science, University of Calgary.

[7] J. Lee, W. Liao, and M. Chen, "A Differentiated Service Model for Enhanced Distributed Channel Access (EDCA) of IEEE 802.11e WLANs", Proceedings of IEEE Globecom 2005.

[8] Cisco Systems, Cisco Aironet 1200 Series Access Point Wireless Quality of Service Deployment Guide, http://www.cisco.com/en/hmpgs/index.html

[9] D. Newman, "Voice over Wireless LAN", Network World online review, January 10, 2005.

[10] D. Pauliukas and V. Vosylius, "Research of Real-Time Traffic Transmission in 802.11 WLANs" Electronics and Electrical Engineering, No. 7, 2005.

[11] C. Williamson, "Internet Traffic Measurement", IEEE Internet Computing, Vol. 5, No. 6, pp. 70-74, November/December 2001.

[12] A. Mahanti, C. Williamson, and M. Arlitt, "Remote Analysis of a Distributed WLAN using Passive Wireless-side Measurement", Performance Evaluation, Vol. 64, No. 9-12, pp. 909-932, October 2007.

[13] WIKIPEDIA. http://en.wikipedia.org/wiki/IEEE_802.11e$\underline{2005}$

[14] IEEE Std 802.11 ${ }^{\mathrm{TM}}-2007$, IEEE Standard for Local and Metropolitan Area Networks - Specific Requirements - Part 11: Wireless LAN Medium Access Control (MAC) and Physical Layer (PHY) Specifications. http://standards.ieee.org/findstds/standard/802.11-2007.html

[15] BackTrack Linux. http://www.backtrack-linux.org/ 